\title{
The Caenorhabditis elegans SOMI-1 zinc finger protein and SWI/SNF promote regulation of development by the mir-84 microRNA
}

\author{
Gabriel D. Hayes, Christian G. Riedel, and Gary Ruvkun ${ }^{1}$ \\ Department of Molecular Biology, Massachusetts General Hospital, Boston, Massachusetts 02114, USA, and Department of \\ Genetics, Harvard Medical School, Boston, Massachusetts 02115, USA
}

\begin{abstract}
Hundreds of microRNAs (miRNAs) have been discovered in metazoans and plants, and understanding of their biogenesis has advanced dramatically; however, relatively little is known about the cofactors necessary for miRNA regulation of target gene expression. In Caenorhabditis elegans, the conserved miRNA let-7 and its paralogs, including mir-84, control the timing of stage-specific developmental events. To identify factors required for the activity of mir-84 and possibly other miRNAs, we screened for mutations that suppress the developmental defects caused by overexpression of mir-84. Mutations in the somi-1 gene prevent these defects without affecting the expression level of mir-84. Loss of somi-1 also causes phenotypes similar to deletion of mir-84, showing that somi-1 is necessary for the normal function of this miRNA. somi-1 encodes a zinc finger protein that localizes to nuclear foci and binds the promoters of let-60/RAS, lin-14, and lin-28, genes that may be targeted by mir-84 and similar miRNAs. Genetic evidence shows that somi-1 inhibits lin-14 and induction of the vulval precursors by the let-60/RAS pathway. Proteomic and genetic screens identified conserved chromatin-remodeling and homeodomain transcription factor complexes that work with somi-1 to regulate differentiation. Our results suggest that somi-1 coordinates a nuclear response that complements the activity of mir-84.
\end{abstract}

[Keywords: microRNAs; mir-84; let-7; SWI/SNF; chromatin; differentiation]

Supplemental material is available for this article.

Received June 3, 2011; revised version accepted August 22, 2011.

microRNAs (miRNAs) are 21 -nucleotide (nt) RNAs found in plants and throughout metazoan phylogeny. The hundreds of known miRNAs are involved in many processes, including timing of developmental events, apoptosis, organ development and function, and many others (for review, see Alvarez-Garcia and Miska 2005).

Animal miRNAs negatively regulate target mRNAs by binding sites in the $3^{\prime}$ untranslated region (UTR), which typically have perfect or near-perfect complementarity to nucleotides 2-8 (the "seed" region) of the miRNA, but contain mismatches to the rest of the miRNA (Wightman et al. 1993; Doench and Sharp 2004). In some cases, miRNAbound mRNAs are directed to cytoplasmic processing bodies to be degraded by nucleases or held intact but their translation inhibited (Bagga et al. 2005; Ding et al. 2005). How the fate of particular mRNAs that are targeted by miRNAs is determined is unknown.

${ }^{1}$ Corresponding author.

E-mail ruvkun@molbio.mgh.harvard.edu.

Article is online at http://www.genesdev.org/cgi/doi/10.1101/gad.17153811.
The first two miRNAs discovered, lin-4 and let-7, act in the heterochronic pathway to control the timing of stagespecific developmental events in Caenorhabditis elegans (Ambros and Horvitz 1984; Lee et al. 1993; Reinhart et al. 2000). lin-4 down-regulates its target genes, lin-14 and lin28 , in early larval development, allowing stage-specific progression in patterns of division of epidermal and vulval precursor cells (VPCs) (Lee et al. 1993; Wightman et al. 1993; Moss et al. 1997). let-7 acts later in larval development to down-regulate target genes such as lin-41 and hbl-1 to allow accumulation of the LIN-29 transcription factor, which directs terminal differentiation of epidermal cells and exit from the molting cycle at the adult stage (Rougvie and Ambros 1995; Reinhart et al. 2000; Slack et al. 2000; Abrahante et al. 2003; Lin et al. 2003).

let- 7 is conserved across bilaterian phylogeny (Pasquinelli et al. 2000), and some targets of this miRNA are also conserved. let-7 regulates orthologs of lin-41 in vertebrate development (Lancman et al. 2005) and the RAS oncogene in worms and humans (Johnson et al. 2005). Likewise, orthologs of lin-4 and let-7 regulate vertebrate orthologs of lin-28. By inhibiting maturation of let-7, Lin28 promotes 
pluripotency of stem cells (for review, see Nimmo and Slack 2009). Conservation of heterochronic miRNAs and their target mRNAs suggests that cofactors for the function of these miRNAs may also be broadly conserved.

Three C. elegans paralogs of let-7-mir-84, mir-48, and mir-241-act with let-7 to control certain developmental events. These miRNAs are identical at the $5^{\prime}$ ends, but diverge at the $3^{\prime}$ ends (Lau et al. 2001). mir-84 is most similar (18 of 22 nt identity) to miR-98 of humans and other vertebrates, shown using ClustalW to align let-7 with 71 of its paralogs in 10 animal species (Supplemental Fig. S1). Human miR-98 targets the HMGA2 oncogene (Hebert et al. 2007). In C. elegans, cessation of molting in adults is directed by let-7 redundantly with its paralogs (Abbott et al. 2005; Hayes et al. 2006). Similarly, all three let-7 paralogs redundantly down-regulate $h b l-1$ during larval development, directing timing of divisions of the VPCs and hypodermal seam cells (Abbott et al. 2005).

While mir-84 acts with its paralogs in these pathways, high gene dosage of mir-84 is sufficient to induce precocious division of the VPCs to cause a penetrant defect in vulval morphogenesis so that animals never lay eggs, which then hatch within the parent (Hayes and Ruvkun 2005; Johnson et al. 2005). To identify factors required for function of mir-84 and potentially other miRNAs, we performed a genetic screen for suppressors of this defect, referred to as Somi (suppressor of overexpressed miRNA) mutants. Here, we describe the somi-1 gene, which encodes a new class of zinc finger protein. In addition to suppressing the phenotypes induced by high mir-84 gene dosage, loss of somi-1 enhances heterochronic phenotypes caused by mutations in let- 7 and its paralogs, showing that somi-1 itself regulates the timing of developmental events. SOMI-1 binds the promoters of particular genes that may be targets of mir-84 and let-7 and copurifies with a SWI/ SNF-like chromatin-remodeling complex that promotes differentiation. Our results suggest that somi-1 coordinates a nuclear response that complements regulation by these miRNAs.

\section{Results}

To explore the function of 1 et-7 and paralogous miRNAs, we generated transgenic strains that overexpress the mir-84 miRNA (Hayes et al. 2006). These animals show precocious development of vulval and epidermal cells similar to lin-14 and other precocious heterochronic mutants (Ambros and Horvitz 1984; Hayes and Ruvkun 2005; Johnson et al. 2005). The VPCs divide precociously in animals bearing the mgIs45[mir-84++] transgene (Supplemental Fig. S2), causing abnormal eversion of the vulva (the Evl phenotype) (Fig. 1A) that prevents all mgIs45-bearing animals from laying eggs (Table 1).

To identify genes required for function of mir-84 (and perhaps other miRNAs), we mutagenized mir-84-overexpressing animals and recovered the very rare eggs laid by the $\mathrm{F}_{2}$ mutant descendants, putative Somi mutants. We focused on the somi-1(mg415) mutant, which strongly suppressed the Evl phenotype. somi-1 mutants appear

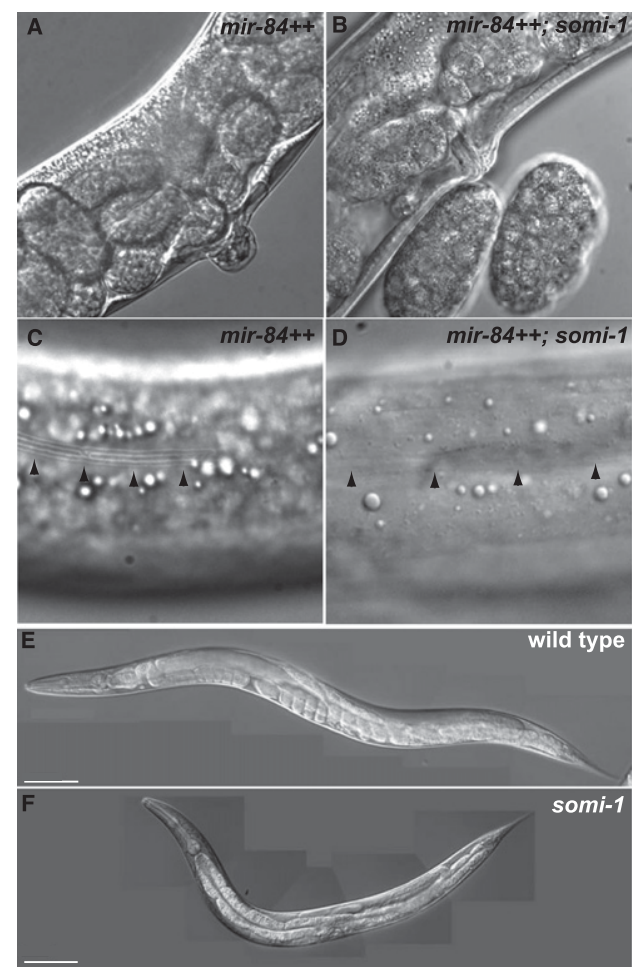

Figure 1. Loss of somi-1 suppresses precocious development caused by overexpression of mir-84. (A) mir-84-overexpressing adult with a vulval protrusion. (B) mir-84-overexpressing somi1(mg415) mutant adult with a functional vulva next to two embryos. (C) Precocious alae (arrowheads) on the cuticle of a mir84-overexpressing L4 larva. (D) A mir-84-overexpressing somi1(mg415) mutant L4 larva lacking alae (arrowheads). Wild-type $(E)$ and somi-1(mg415) (F) young adults $74 \mathrm{~h}$ after egg lay at $20^{\circ} \mathrm{C}$. Bars, $100 \mu \mathrm{m}$.

superficially wild type, except that adults have slightly reduced body length, $\sim 12 \%$ shorter in young adults (Fig. $1 \mathrm{E}, \mathrm{F})$. Genetic mapping (see the Supplemental Material) placed mg415 within a 1.9-map unit region of chromosome five. RNAi against one gene of the 412 tested in this interval, M04G12.4, enabled mgIs45-bearing animals to lay eggs. Sequencing of M04G12.4 in the mg415 mutant revealed a single cytosine-to-thymine transition (Fig. 2A) predicted to cause a glutamine to an ochre nonsense codon substitution (Fig. 2B). Sequencing of other Somi mutants revealed that two of them, mg431 and mg432, bear distinct nonsense mutations in somi-1 (Fig. 2).

All three alleles of somi-1 strongly suppressed the Evl phenotype when they were outcrossed to wild type and introduced to a strain bearing an unmutagenized copy of mgIs45 (Fig. 1A,B; Table 1). Similar results were obtained for a deletion mutation in M04G12.4 (tm562) that eliminates 530 base pairs (bp) encoding the final third of exon two and the following splice donor site. A transgene bearing the M04G12.4 ORF restored the Evl phenotype to mgIs 45; somi-1(mg415) animals (90\%, $n=$ 49) compared with siblings lacking the transgene $16 \%$, $n=17$ ), showing that somi-1 is encoded by M04G12.4 (Table 1). 
Table 1. Loss of somi-1 suppresses the precocious development caused by overexpression of mir-84

\begin{tabular}{|c|c|c|c|}
\hline Genotype & $\begin{array}{c}\text { Evl } \\
{[\%(\mathrm{n})]}\end{array}$ & $\begin{array}{l}\text { Alae formation at L4 } \\
\text { stage }(\%[\mathrm{n}])^{\mathrm{c}}\end{array}$ & $\begin{array}{l}\text { Alae formation at adult } \\
\text { stage }(\%[\mathrm{n}])^{\mathrm{c}}\end{array}$ \\
\hline Wild type & $0 \%(20)$ & $0 \%(10)$ & $100 \%(23)$ \\
\hline mgIs45[mir-84++] & $100 \%(22)^{a}$ & $70 \%(30)^{\mathrm{a}}$ & nd \\
\hline $\operatorname{mgIs} 45 /+$ & $81 \%(27)$ & nd & nd \\
\hline somi-1(mg415) & $0 \%(20)$ & $0 \%(10)$ & $100 \%(22)$ \\
\hline mgIs45; somi-1(mg415) & $10 \%(39)^{\mathrm{a}}$ & $0 \%(21)$ & $100 \%(11)$ \\
\hline mgIs 45 ; somi-1(mg431) ${ }^{\mathrm{d}}$ & $20 \%(50)^{\mathrm{a}}$ & nd & nd \\
\hline mgIs 45 ; somi-1(mg432) ${ }^{\mathrm{d}}$ & $24 \%(45)^{\mathrm{a}}$ & nd & nd \\
\hline mgIs45; somi-1(tm562) & $9 \%(45)^{\mathrm{a}}$ & $18 \%(11)^{\mathrm{b}}$ & $100 \%(12)$ \\
\hline mgIs 45; control RNAi & $100 \%(103)$ & nd & nd \\
\hline mgIs 45; somi-1 RNAi & $36 \%(74)^{a}$ & nd & nd \\
\hline mgIs 45; somi-1(mg415) & $6 \%(17)$ & $28 \%(18)$ & nd \\
\hline mgIs 45; somi-1(mg415); mgEx740[somi-1(+)] $]^{\mathrm{e}}$ & $90 \%(49)^{\mathrm{a}}$ & $75 \%(8)^{\mathrm{b}}$ & nd \\
\hline mgIs 45; somi-1(mg415) ${ }^{\mathrm{e}}$ & $6 \%(17)$ & $17 \%(12)$ & $100 \%(9)$ \\
\hline mgIs45; somi-1(mg415); mgEx739[SOMI-1::GFP] & $70 \%(27)^{\mathrm{a}}$ & $73 \%(11)^{\mathrm{b}}$ & $100 \%(6)$ \\
\hline
\end{tabular}

(nd) Not determined.

${ }^{a} P<0.0001$ compared with control, Fisher's exact test, two-tailed.

${ }^{\mathrm{b}} P<0.05$ compared with control, Fisher's exact test, two-tailed.

${ }^{\mathrm{c}}$ One side of each animal was examined by Nomarski optics, and the percentage of animals bearing complete or partial alae is indicated.

$\mathrm{d}_{\text {somi-1 }}$ was marked with wIs54[scm::gfp] in these strains.

eSiblings that either carried or lacked the transgenes encoding somi-1 (mgEx740 or mgEx739) were scored.

somi-1 encodes a zinc finger protein with predicted nuclear localization

somi-1 encodes two differentially spliced $\mathrm{C}_{2} \mathrm{H}_{2}$-type zinc finger isoforms (Fig. 2A), with one lacking the $\mathrm{N}$ terminus encoded by the first exon. SOMI-1 is conserved in the nematodes Caenorhabditis briggsae and Caenorhabditis remanei (Fig. 2B), but database searches reveal no orthologs in organisms other than nematodes, although members of the zinc finger class of nucleic acid-binding proteins exist across phylogeny. The three nonsense mutations we identified in somi-1 are predicted to cause severely truncated SOMI-1 proteins lacking the zinc finger domain and nuclear localization signal (NLS), and thus are likely null alleles.

As a zinc finger protein, SOMI- 1 is predicted to bind nucleic acids (Laity et al. 2001). We found a single NLS in SOMI-1 (Fig. 2B), consistent with its nuclear localization (see below) and suggesting that it may regulate transcription or other nucleic acid processing mechanisms. In addition to the zinc finger domain, a similar sequence lacks the second histidine residue of a canonical zinc finger (Fig. 2B). Seven consecutive proline residues at the $\mathrm{C}$ terminus are perfectly conserved in C. briggsae and C. remanei.

somi-1 facilitates mir-84 function in differentiation of the hypodermis

Loss of somi-1 suppressed not only precocious vulval development caused by mir-84 overexpression, but also precocious appearance of alae, cuticular structures secreted by the hypodermal seam cells, which normally form in adults. Animals bearing $m g I s 45$ formed alae precociously, at the third larval molt $(70 \%, n=30)$, but this defect was fully suppressed by the somi-1(mg415) mutation $(n=21)$ (Fig. 1C,D; Table 1).

mir-84 and its paralog, let-7, together direct terminal differentiation of the seam cells and exit from the molting cycle (Hayes et al. 2006). Animals bearing the let-7(mg279) mutation, a weak allele that reduces abundance of let-7 RNA (Bracht et al. 2004), can fail to exit the molting cycle at the adult stage and instead enter a supernumerary molt. Loss of mir-84 enhances the penetrance of let-7(mg279) supernumerary molting (Hayes et al. 2006). We reasoned that if somi-1 facilitates function of mir-84 in the hypodermis, then loss of somi-1 should also enhance let-7(mg279). We assayed supernumerary expression of a green fluorescent protein (GFP) reporter, mlt-10p::gfp-pest, which peaks in expression before each of the larval molts and is not expressed in wild-type adults (Frand et al. 2005). When somi-1(mg415); let-7(mg279) double and let-7(mg279) single mutants were grown in synchrony, a much higher percentage of the double mutants expressed mlt-10p::gfppest at $72 \mathrm{~h}$ of growth (Fig. 3A,B). let-7 mir-84 double mutant adults similarly express mlt-10::gfp sooner after the fourth molt than let-7(mg279) single mutants (Supplemental Fig. S3; Hayes et al. 2006). We obtained similar results when somi-1 was inactivated by RNAi in a let7(mg279) mutant, but did not see supernumerary expression of mlt-10p::gfp-pest in wild-type or mir-84 single mutant animals in which somi-1 was inactivated (Supplemental Fig. S3) or in somi-1 single mutants (Fig. 3A). Consistent with these results, inactivation of somi-1 enhanced the lethality of a let-7(n2853) point mutant (Table 2). These results suggest that somi-1 and mir-84, together with let-7, direct terminal differentiation of the seam cells, exit from the molting cycle, and formation of the vulva. Furthermore, somi-1 is not merely required for phenotypes induced by expression of mir-84 from a transgene, but facilitates activity of the endogenous miRNA.

mir-48 and mir-241 act with mir-84 to control larval divisions of the seam cells and exit from the molting cycle in adults (Abbott et al. 2005). Supernumerary expression of mlt-10p::gfp-pest in mir-48 mir-241 adults began earlier 
Hayes et al.

A

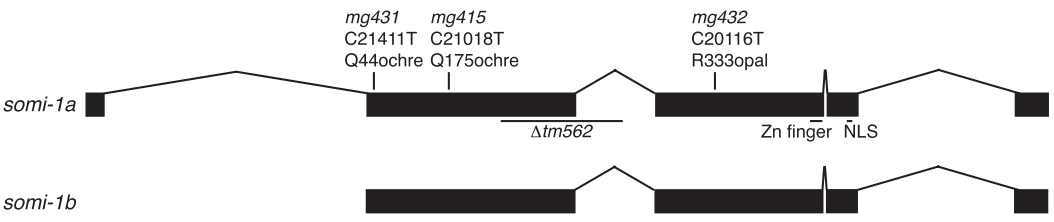

B

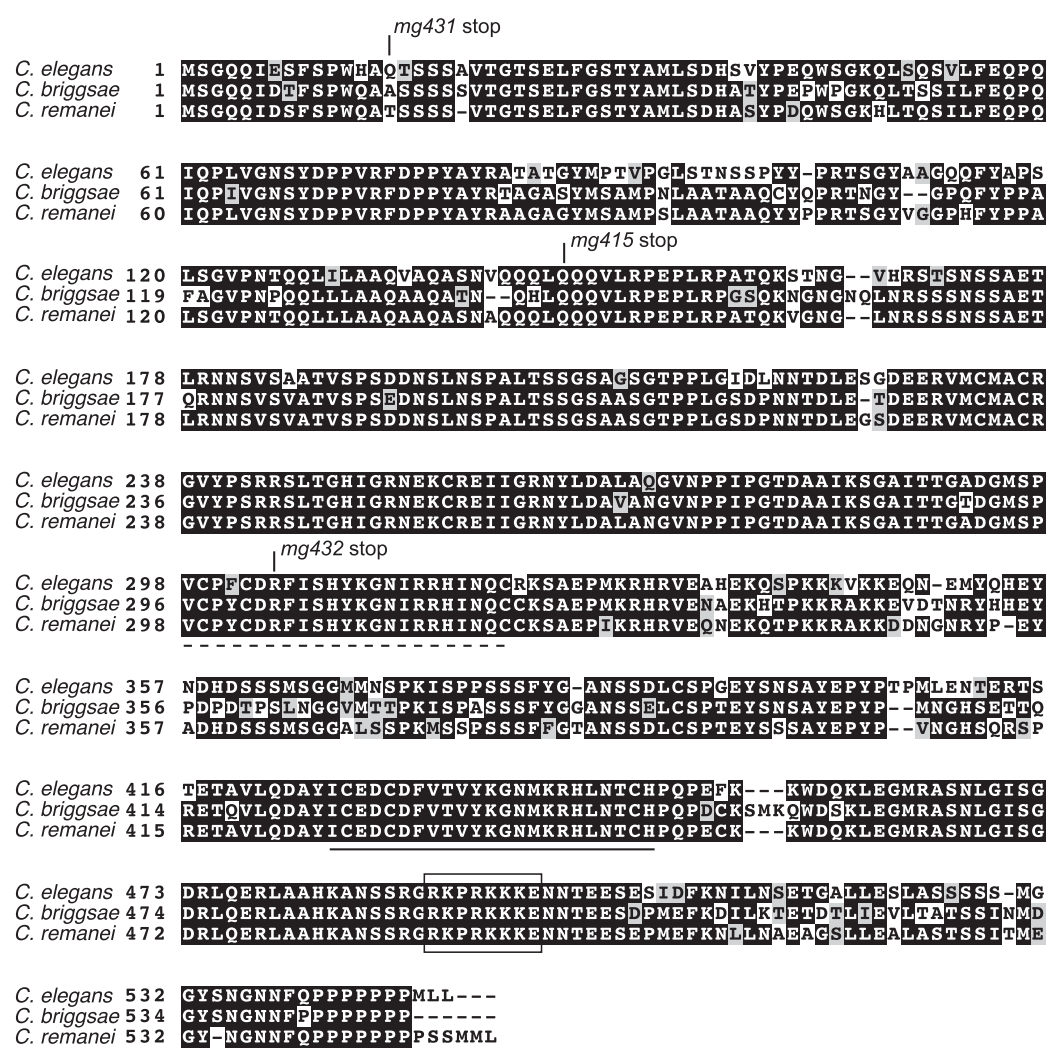

Figure 2. somi-1 encodes two $\mathrm{C}_{2} \mathrm{H}_{2}$ zinc finger proteins that are conserved in nematodes. (A) Gene structures for somi-1a and somi-1b isoforms, which are supported by expressed sequence tag data (not shown). Boxes denote exons and lines denote introns. Positions of mutations in somi-1, the zinc finger domain, and predicted NLS are shown. Nucleotide numbering refers to cosmid M04G12.4 (accession no.: emb|Z88103) and amino acid numbering refers to M04G12.4A (accession no.: ref|NP_506320.3). (B) Alignment of SOMI-1B sequences from C. elegans and two related nematodes. Black shading denotes amino acid identity and gray shading denotes similarity. The zinc finger domain is underlined, and a similar sequence that lacks the second histidine residue is marked with a dashed line. The NLS is boxed, and positions where the protein is truncated in somi1 nonsense mutants are marked by vertical lines. The alignment was produced with ClustalW 1.83 and shading was produced with Boxshade 3.21. Accession numbers are as follows: C. elegans: ref|NP_872161.2|; C. briggsae: ref|XP_002647630.1; and C. remanei: ref|XP_003114157.1. when somi-1 was inactivated by RNAi compared with animals exposed to dsRNA homologous to no worm gene (hereafter, control RNAi) (Fig. 3C). Animals lacking all three of these let-7 paralogs often burst at the last larval molt, similar to let-7-null mutants (Abbott et al. 2005). somi-1(RNAi) enhanced this lethality $(43 \%, n=273)$ in mir48 mir-241 mutants compared with control RNAi $(27 \%, n=$ 268). Thus, somi-1 acts with mir-48 and mir-241 to promote stability of the vulva and exit from the molting cycle, potentially by facilitating function of mir-84.

To examine the role of somi-1 in miRNA-regulated temporal specification of the hypodermis, we observed expression of col-19::gfp (Abrahante et al. 1998). Synthesis of the adult-specific collagen COL-19 is directed by the LIN-29 transcription factor (Rougvie and Ambros 1995), and let-7 acts with its paralogs to promote full expression of col-19::gfp in the hypodermal syncytium and seam cells (Abbott et al. 2005; Hayes et al. 2006). Gravid let-7(mg279) mutants in which somi-1 was inactivated by RNAi expressed col-19::gfp in the seam cells but not the hypodermis (Fig. 3D), a phenotype similar to let-7(mg279) mir84(tm1304) double mutants (Hayes et al. 2006). In contrast, the majority of let-7 mutants exposed to control
RNAi expressed GFP in both the seam cells and hypodermis. Wild-type animals nearly all had full expression of col-19::gfp even upon RNAi against somi-1. These results confirm that somi-1 acts in the heterochronic pathway to specify developmental timing of the hypodermis.

Finally, we asked whether somi-1 is required for precocious development caused by overexpression of other let7 family miRNAs. We expected loss of somi-1 to suppress the vulval defect caused by overexpression of mir-48 (Li et al. 2005), which is similar to that caused by overexpression of mir-84; however, worms bearing the veIs48 [mir-48(ve33)] transgene had a completely penetrant Evl phenotype even when somi-1 was inactivated by mutation or RNAi (Supplemental Table S1). In contrast, only 7\% ( $n=$ 83) of animals bearing a transgene that drives precocious expression of let-7 under the lin-4 promoter (Hayes and Ruvkun 2006) expressed col-19::gfp at the L4 stage when fed somi-1(RNAi) compared with $48 \%(n=177)$ of animals fed control RNAi (Supplemental Table S1). This suggests that function of mir-48 is not mediated by somi-1 and that somi-1 may promote inhibition of one or more targets that are specific to mir-84 and let-7 but not mir-48, at least when each miRNA is overexpressed. 


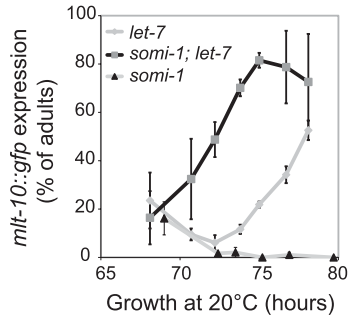

c

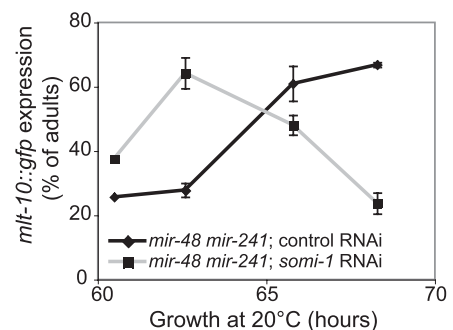

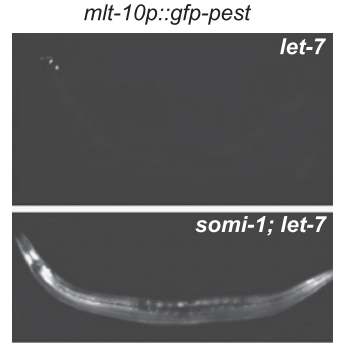

D

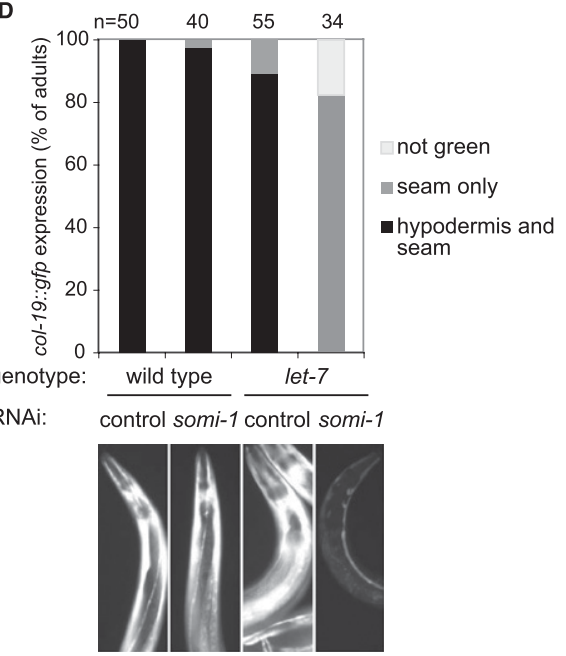

Figure 3. Loss of somi-1 enhances defects in exit from the molting cycle and differentiation of the hypodermis in adults with mutations in let-7 or its paralogs. (A) Supernumerary expression of mlt-10p::gfp-pest, a reporter for molting, in populations of GR1436, GR1605, and GR1707 mutant adults plated as synchronized L1 larvae. The combined results of two experiments are shown. A total of at least 90 animals were scored at each time point. $(A, C)$ Error bars indicate SEM. $(B)$ Typical mlt-10p::gfp-pest expression in let-7(mg279) single and somi-1(mg415); let-7(mg279) double mutants at $74.5 \mathrm{~h}$ of growth. (C) mlt-10p::gfp-pest expression in mir-48 mir-241(nDf51) mutant adults plated as synchronized L1 larvae to somi-1 or control RNAi. The combined results of three experiments are shown. A total of at least 40 animals were scored at each time point. $(D)$ Loss of somi-1 enhances the failure of let-7(mg279) mutants to express a reporter for the adult-specific collagen COL-19 in the hypodermal syncytium. Animals were fed somi-1 or control RNAi, and their progeny were scored as gravid adults for expression of col-19::gfp using a Zeiss SV-6 microscope. The combined results of two independent experiments are presented. $(B, D)$ Images of typical animals were captured using the same exposure settings and were processed identically.
Many protein-coding genes in the heterochronic pathway bear potential target sites for let-7 family miRNAs (Reinhart et al. 2000), and inactivation of some of these genes causes precocious developmental phenotypes like those caused by overexpression of mir-84 (Ambros and Horvitz 1984). The lin-14 3' UTR bears several potential targets for mir-84 (Hayes and Ruvkun 2005), and expression of mir-84 begins in the early L1 stage (Esquela-Kerscher et al. 2005; Hayes et al. 2006), suggesting that mir-84 may act with lin-4 during early larval development to regulate lin-14. Indeed, elevated expression of mir-84 inhibits lin-14 in a 3' UTR-dependent way (Hayes and Ruvkun 2005). The lin-14(n360) mutation, which prevents synthesis of LIN-14B but leaves LIN-14A unaffected, causes precocious formation of alae. This defect is suppressed when LIN-14A levels are elevated by loss of the lin-4 miRNA (Reinhart and Ruvkun 2001), suggesting loss of somi-1 could similarly raise expression of particular heterochronic genes. Indeed, only half of lin-14(n360) mutants formed alae at the L4 stage when somi-1 was inactivated, in contrast to all lin14(n360) mutants fed control RNAi (Supplemental Table S1). Similarly, the Evl phenotype was less penetrant upon inactivation of lin-14 in somi-1 mutants than in wild type (Supplemental Table S1). Thus, loss of somi-1 may suppress the effects of overexpression of mir-84 at least in part by increasing expression of lin-14. In contrast, loss of somi-1 did not suppress the Evl phenotype of a temperaturesensitive allele of lin-14, and RNAi against other heterochronic genes caused similarly penetrant precocious phenotypes in wild-type and somi-1 mutant animals.

\section{somi-1 is not required for expression of mir-84}

Loss of somi-1 could suppress mir-84 overexpression phenotypes and phenocopy a mir-84-null mutant by reducing expression of mir-84. All animals with two copies of the mgIs45[mir-84++] transgene $(n=22)$ but only $81 \%$ $(n=27)$ of animals with one copy of this transgene formed a vulval protrusion that prevented egg laying (Table 1), suggesting that loss of half of the expression of mir-84 from mgIs45 can only weakly suppress precocious vulval development. Northern blotting showed that loss of somi-1 did not alter the level of endogenous mir-84 in two independent samples (Supplemental Fig. S4A), and expression of mir-84 from mgIs 45 in a somi- 1 mutant was similar to wild type in two cases and decreased by $44 \%$ in one case (Supplemental Fig. S4B). Thus, change in mir-84 abundance

Table 2. Loss of somi-1 enhances the lethality of a let-7 point mutant

\begin{tabular}{llc}
\hline Genotype & RNAi & Lethality $(\%[\mathrm{n}])$ \\
\hline Wild type & Control & $0 \%(95)$ \\
Wild type & somi-1 & $0.6 \%(172)$ \\
let-7(n2853) & Control & $4 \%(566)$ \\
let-7(n2853) & somi-1 & $81 \%(505)^{\mathrm{a}}$ \\
\hline
\end{tabular}

Wild-type or GR1434 L4 animals were placed on bacteria expressing dsRNA homologous to the indicated gene or no worm gene, and their progeny were scored for bursting at the L4-to-adult transition.

${ }^{\mathrm{a}} P<0.0001$ compared with control, Fisher's exact test, two-tailed. 
in total RNA was too small to account for the nearly complete suppression by loss of somi-1 of the vulva formation defect caused by mir-84 overexpression. Variation in expression of mir- 84 from the transgene could be due to spontaneous partial silencing of the transgene, a process we do not believe somi-1 is involved in. Northern analysis cannot exclude the possibility that somi-1 is required for mir-84 expression in particular tissues. However, we found no change in expression of mir-84::gfp (Hayes et al. 2006) in a somi-1 mutant (data not shown). These results suggest that somi-1 does not regulate expression of mir-84, but acts genetically downstream from mir-84.

\section{SOMI-1 is localized to nuclear foci and coexpressed} with mir-84

To determine in which tissues somi-1 acts, we generated transgenes that fuse sequence $5^{\prime}$ of the transcriptional start of somi-1a to GFP. The somi-1p::gfp transcriptional fusion gene was expressed in the hypodermal seam cells (Fig. 4A) and the somatic gonad and VPCs (Fig. 4B). This expression pattern is similar to that of reporters for mir-84 (Esquela-Kerscher et al. 2005; Johnson et al. 2005; Hayes et al. 2006) and shows that somi-1 is expressed in tissues that develop precociously when mir-84 is overexpressed. Seam cell expression of somi-1p::gfp was most common in L4 larvae and adults, suggesting that somi-1 is up-regulated upon differentiation of this tissue (data not shown). The earliest somi-1p::gfp expression was in comma stage embryos (Fig. 4C), whereas mir-84::gfp expression was first detected in larvae (Hayes et al. 2006). somi-1p::gfp was also expressed in body wall muscle (Fig. 4A) and certain neurons in the head (Fig. 4D) and tail (data not shown), which do not express mir-84::gfp. The partial overlap in spatial and temporal expression patterns of GFP reporters for somi-1 and mir-84 is consistent with somi-1 not only facilitating function of mir-84, but also acting independently of mir-84.

Immunofluorescence using antisera raised against a full-length His-SOMI-1A fusion protein confirmed the expression pattern revealed by somi-1p::gfp, except that hypodermal expression of SOMI-1 was more evident by immunostaining. SOMI-1 was detected in the nuclei of embryos beginning at the comma stage and in larvae of all stages and adults in hypodermal, body wall muscle, and most other cells in wild type (Fig. 4E) but not in a somi1(mg415) mutant (Fig. 4F). SOMI-1 was also detected in the somatic gonad, including the distal tip (Fig. 4G) and sheath cells (data not shown).

Fusion of the somi-1 promoter and genomic proteincoding sequence to the $\mathrm{N}$ terminus of GFP yielded a rescuing SOMI-1::GFP reporter (Table 1) that was typically localized to the nucleus but excluded from the nucleolus. SOMI-1::GFP was often concentrated in nuclear foci, which were most apparent in embryos (Fig. 5A), but could also be detected in larvae (Fig. 5B). SOMI-1 immunofluorescence also concentrated in nuclear foci and colocalized extensively with DNA, as visualized by staining with diamino-2-phenylindole (DAPI) (Fig. 5C). SOMI-1::GFP was expressed in the same tissues as the
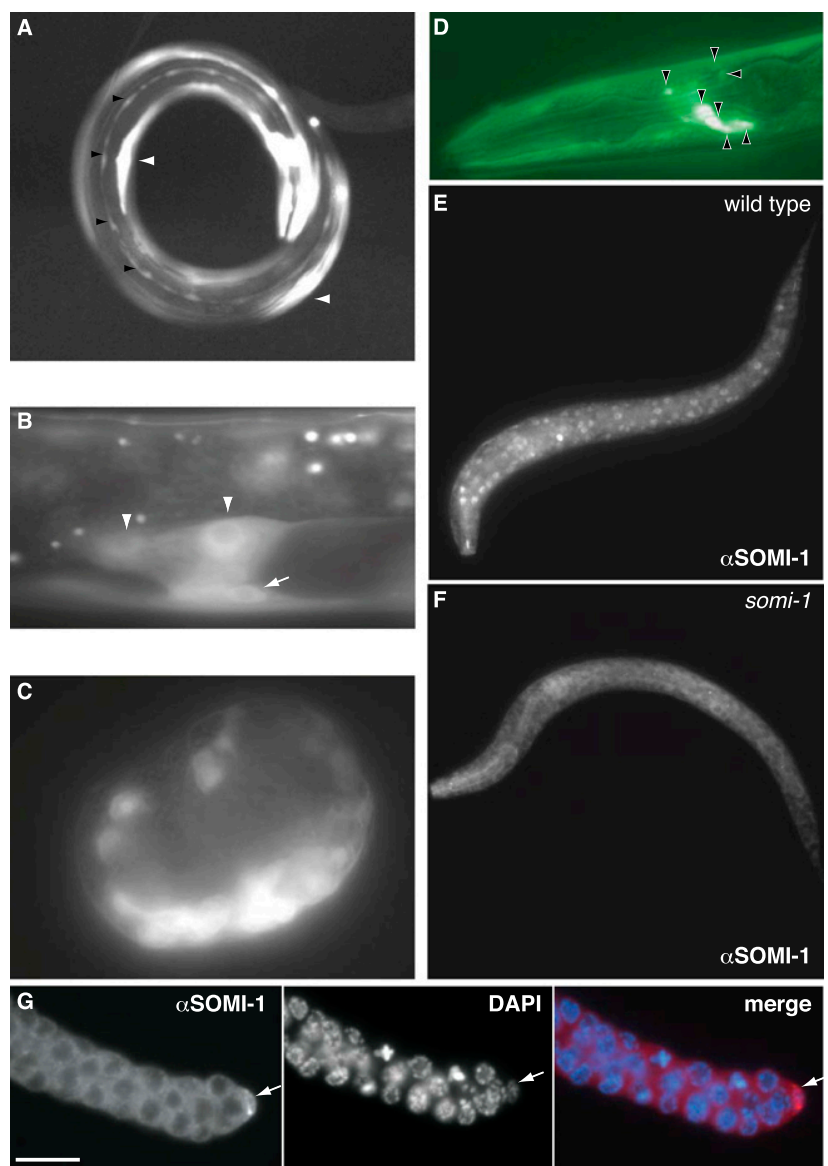

Figure 4. somi-1 is expressed in tissues affected by loss of paralogs of let-7 and in other tissues. $(A-D)$ somi-1p::gfp::somi$13^{\prime}$ UTR expression. (A) Adult expressing somi-1p::gfp in the hypodermal seam cells (black arrowheads), body wall muscle (white arrowheads), and neurons. (B) somi-1p::gfp in the somatic gonad (arrowheads) and a VPC (arrow) of an L3 larva. (C) Onset of somi-1p::gfp expression in a comma stage embryo. (D) somi-1p:: $g f p$ in head neurons (arrowheads) of an L3 larva. GFP image overlaid on Nomarski image. (E) SOMI-1 immunostaining in the nuclei of hypodermal, muscle, and other cells of a wild-type L1 larva. (F) Immunostaining of a somi-1(mg415) mutant L1 larva showing only background staining. $(G)$ SOMI-1 immunostaining in the distal tip cell (arrow) of the somatic gonad. Faint cytoplasmic staining of the germ cells was sometimes also seen in somi-1 mutants and may be nonspecific. Bar, $10 \mu \mathrm{m}$.

transcriptional somi-1p::gfp fusion gene as well as the hypodermal syncytium and gut (data not shown). This expression pattern matches SOMI-1 immunostaining and suggests that enhancers embedded downstream from the start codon that are absent from somi-1p::gfp drive expression of somi-1 in some tissues.

\section{SOMI-1 binds the promoters of particular heterochronic genes and let-60/RAS}

Given the localization of SOMI-1 to nuclear foci, we expected that somi-1 might bind the promoters of particular heterochronic genes or others involved in development of the vulva and hypodermis. To test this, we performed 

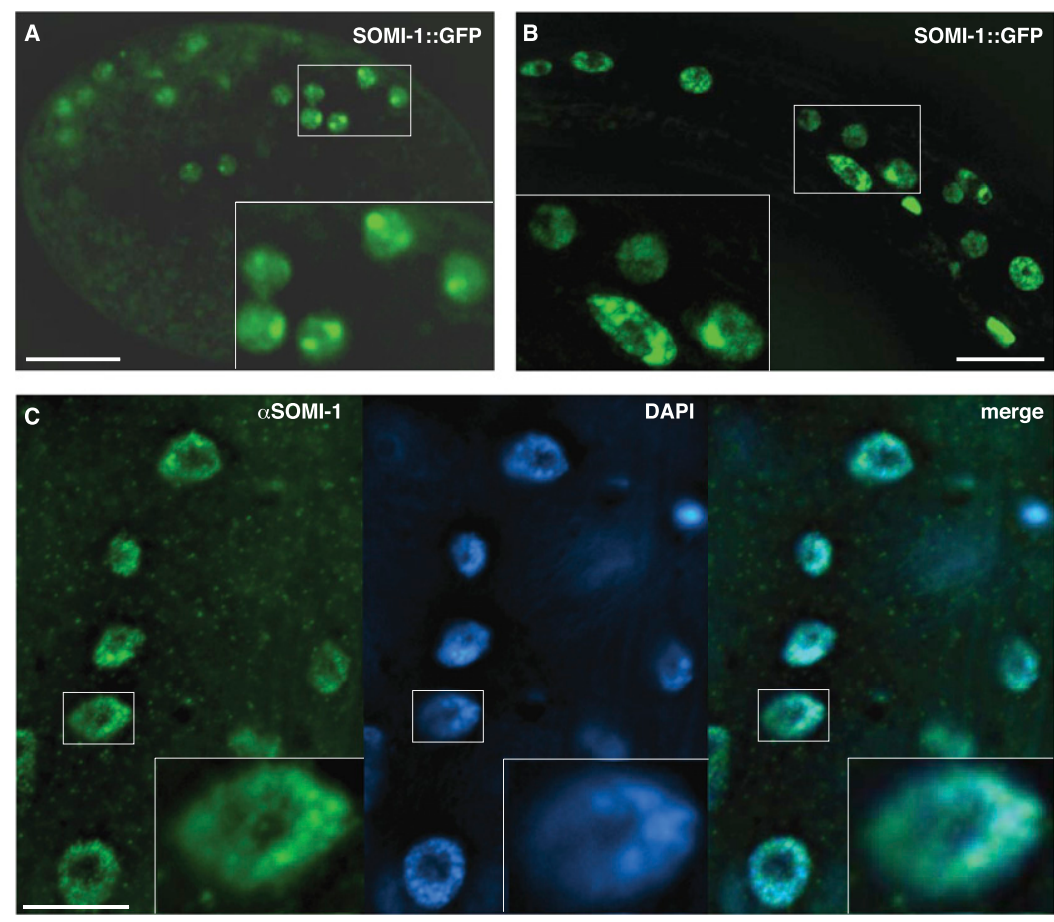

Figure 5. SOMI-1 localizes to nuclear foci. $(A, B)$ mgIs57[SOMI-1::GFP] expression. (A) SOMI-1:: GFP in the nuclei of a comma stage embryo. The image corresponds to one deconvolved optical section. (B) SOMI-1::GFP in hypodermal and neuronal nuclei of an L2 larva. Five deconvolved optical sections were flattened into one image. (C) SOMI-1 immunostaining (green) in hypodermal nuclei near the tail of a wild-type adult with DNA visualized by DAPI. Images correspond to one deconvolved optical section. $(A-C)$ Bars, $10 \mu \mathrm{m}$. Boxed areas are magnified in insets. chromatin immunoprecipitation (ChIP) on animals bearing SOMI-1::GFP. The promoter of let-60/RAS was enriched up to 4.6-fold over an intergenic control and that of lin-28 was enriched up to 3.2-fold in two independent experiments on L2 larvae (Fig. 6A; Supplemental Fig. S5B). The lin-14 $a$ and lin-14b promoters were enriched variably in L2 animals (1.5-fold to 3.1-fold), but lin-14a, lin-28, and let-60 promoters were enriched more than fourfold in ChIP performed on a mixture of gravid adults and embryos (Fig. 6A; Supplemental Fig. S5). We observed no or inconsistent enrichment of the promoters of let-7, mir-84, or other heterochronic or EGF/RAS pathway genes (lin-45/ $R A F$ and let-23/EGF-R), or those of selected transcription factors involved in vulval development, such as egl-5 and egl-13 (Fig. 6A; Supplemental Fig. S5).

\section{somi-1 facilitates regulation of let-60/RAS by mir-84}

mir-84 regulates the C. elegans gene let-60, and let-7 regulates its human homolog, the oncogene RAS (Johnson et al. 2005). Activating mutations in let-60 cause elevated induction of the VPCs, resulting in the appearance of ventral protrusions, the multivulva (Muv) phenotype (Beitel et al. 1990). let-60(n1046) mutants in which somi-1 was inactivated by RNAi formed an average of $2.3 \pm 1.0$ ( $n=$ $214)$ ventral protrusions compared with $1.9 \pm 1.0(n=221)$ for animals on control RNAi $(P<0.0001$, Student's $t$-test $)$, consistent with somi-1 facilitating down-regulation of let60 (Fig. 6B). somi-1(RNAi) reduced 3.1-fold the percentage of adults with no ventral protrusions and increased 3.4-fold the percentage of adults with four protrusions compared with let-60(n1046) mutants treated with control RNAi $(P<$ 0.01 , Fisher's exact test). In contrast, somi-1(RNAi) in a mir-84-null mutant background had no effect on the number of ventral protrusions in let-60(n1046). Finally, the mean number of ventral protrusions in $n 1046 \mathrm{mu}-$ tants that overexpressed mir-84 increased from $1.5 \pm 1.0$ $(n=146)$ to $2.1 \pm 1.0(n=139)$ when somi-1 was inactivated. The percentage of mir-84++; let-60(gf) animals with no protrusions was reduced 2.5 -fold and the percentage with three protrusions was increased 2.6-fold by inactivation of somi-1 $(P<0.01$, Fisher's exact test). These data are consistent with mir-84 negatively regulating let60/RAS in a somi-1-dependent way. Together with ChIP data showing binding of SOMI-1 to the let- 60 promoter, they suggest that somi-1 inhibits expression of let-60 in VPCs that do not normally adopt vulval fates. The fact that deletion of mir-84 abolishes enhancement of let-60(gf) by loss of somi-1 suggests that somi-1 mediates a nuclear response to the activity of mir-84. Given that SOMI-1:: GFP was not enriched at the promoter of mir-84 and loss of somi-1 did not appear to change expression of reporters for mir-84 in the VPCs (data not shown), somi-1 is more likely to mediate response to, rather than expression of, mir-84.

Suppression of a lin-14(1f) or enhancement of a let-60(gf) mutation by loss of somi-1 and the binding of SOMI-1:: GFP to the promoters of these genes suggest that they may be up-regulated in a somi-1 mutant. However, quantitative real-time RT-PCR (qRT-PCR) analysis of total RNA from synchronously grown animals at various stages did not reveal up-regulation of $\operatorname{lin}-14$, let-60, or other potential targets of mir-84. Indeed, these transcripts typically had slightly (approximately twofold) reduced abundance in a somi-1 mutant relative to wild type (data not shown). A let-60p::gfp::1et-60 3' UTR reporter also did not show upregulation in the VPCs of a somi-1 mutant, although the intense, ubiquitous expression of let-60::gfp could mask such a change (data not shown). Staining with LIN-14 
A

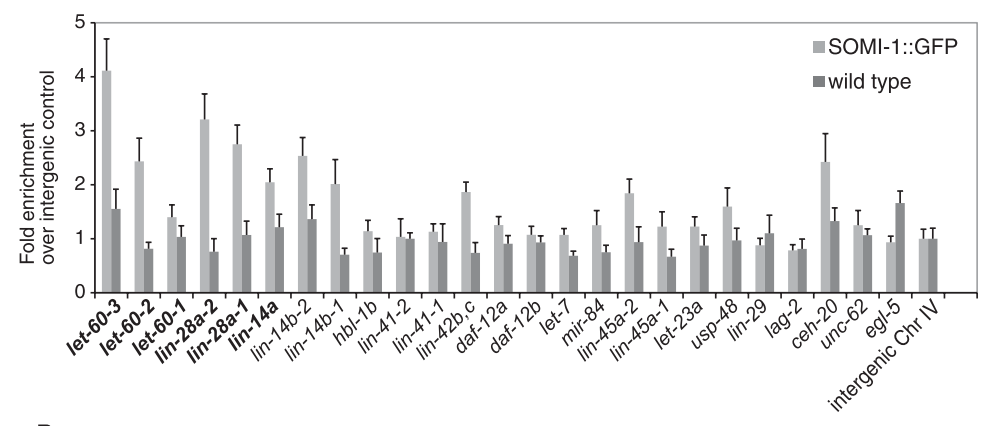

B

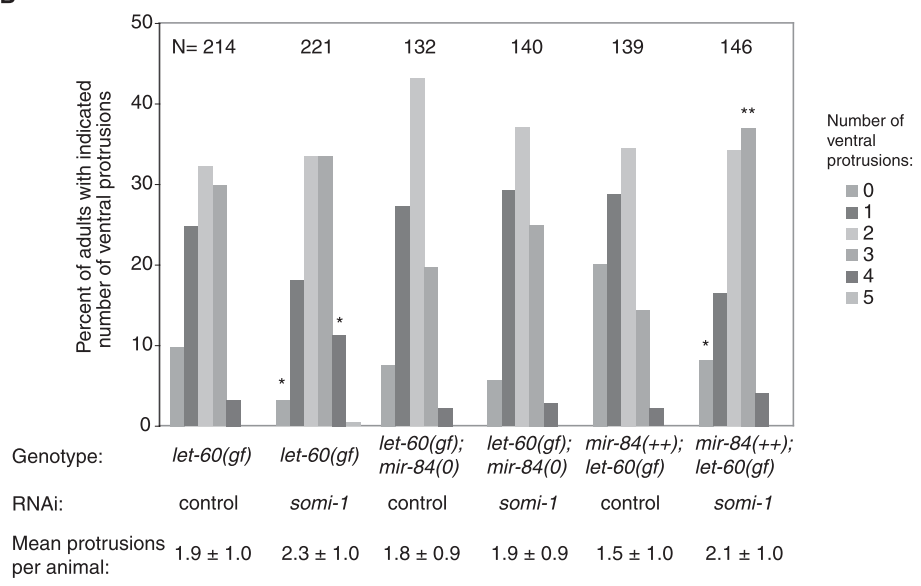

Figure 6. SOMI-1 is enriched at the promoters of let60/RAS, lin-14, and lin-28. (A) Chromatin was isolated from $\alpha$-GFP immunoprecipitates of L2 stage SOMI-1:: GFP or wild-type worms and the purified DNA was assayed by qPCR with primers homologous to the promoters of candidate genes (see Supplemental Table S5). The abundance of each DNA relative to an intergenic control is plotted. Genes in bold were consistently enriched at least twofold in independent experiments. Error bars indicate SEM in qPCR replicates. $(B)$ Inactivation of somi-1 enhances a let-60 gain-of-function mutant. MT2124, GR1689, or GR1690 animals were placed on somi-1(RNAi) or control bacteria, and the number of ventral protrusions, representing ectopic induction of the VPCs, was scored in each of their adult progeny using a dissecting microscope. The mean number of protrusions per animal \pm SD and the percentage of animals with the indicated number of protrusions are indicated. Significant differences compared with control are indicated: $\left(^{\star}\right)$ $P<0.01 ;\left(^{\star \star}\right) P=0.0001$, Fisher's exact test. antibody was also not obviously changed in somi-1 mutants (data not shown). Mutation of somi-1 may cause changes in gene expression too subtle to detect with current methods that nonetheless cause phenotypic change. Alternatively, rather than affecting transcription, somi-1 could promote targeting of transcripts for miRNA-mediated silencing upon export to the cytoplasm. Supporting this possibility, the promoter from which an mRNA is transcribed can affect its regulation by miRNAs (Kong et al. 2008).

\section{SOMI-1 copurifies with SWI/SNF-like proteins that promote miRNA-mediated differentiation}

To examine how somi-1 acts with the mir-84 miRNA, we looked for proteins that bind to SOMI-1. Functional SOMI$1::$ GFP was purified from mixed stage extracts by immunoprecipitation followed by identification of copurifying proteins (Supplemental Fig. S6A) by mass spectrometry (LC-MS/MS). This yielded several specific binding partners (Supplemental Table S2), although most were low in abundance, suggesting that they bind transiently, only in particular tissues, or at certain developmental stages.

We used RNAi tests to evaluate the functional importance of any specific binding partners. Because depletion of some interactors, such as SWSN-1 (Cui et al. 2004), causes an Evl phenotype in a wild-type background that could complicate interpretation of vulval phenotypes, we used hypodermal assays. We reasoned that reduced abundance of proteins that promote function of SOMI-1 should phenocopy somi-1, enhancing a let-7(mg279) mutant, whereas depletion of proteins that oppose function of SOMI-1 might suppress a somi-1; let-7 double mutant. Inactivation of several candidates significantly increased the percentage of synchronously growing let-7(mg279) worms that showed supernumerary expression of mlt-10::gfp or lacked expression of col-19::gfp (Fig. 7B; Supplemental Table S2; Supplemental Fig. S7A). Most of the gene inactivations that enhanced let-7 in both assays correspond to components of a chromatin-remodeling complex similar to yeast SWI/ SNF or RSC and human BAF and PBAF, respectively (Fig. 7A; Supplemental Table S2). Functions of other categories of SOMI-1 interactors will be described separately. Depletion of the majority of SWI/SNF components for which RNAi clones were available enhanced let-7(mg279) in both assays (Fig. 7A). We did not observe supernumerary expression of mlt-10::gfp in wild-type animals treated with the RNAi clones we assayed (data not shown). Only swsn-1 RNAi blocked expression of col-19::gfp in the majority of wild-type gravid adults (Supplemental Table S2; Supplemental Fig. S7B). This homolog of BAF155, a core component of $\mathrm{BAF}$ and PBAF, may be especially sensitive to depletion.

To further examine the genetic interaction between SWI/ SNF and let-7, we observed mlt-10::gfp expression over time in mutants grown on the RNAi clones that caused the most penetrant defects in the mlt-10::gfp assay. swsn-2.1 and tag-298 encode orthologs of accessory components that promote the function of SWI/SNF complexes and their recruitment to particular loci (Kwon and Wagner 2007; Moshkin et al. 2007). Inactivation of swsn-2.1 and tag-298, like somi-1(RNAi), caused earlier onset of supernumerary 


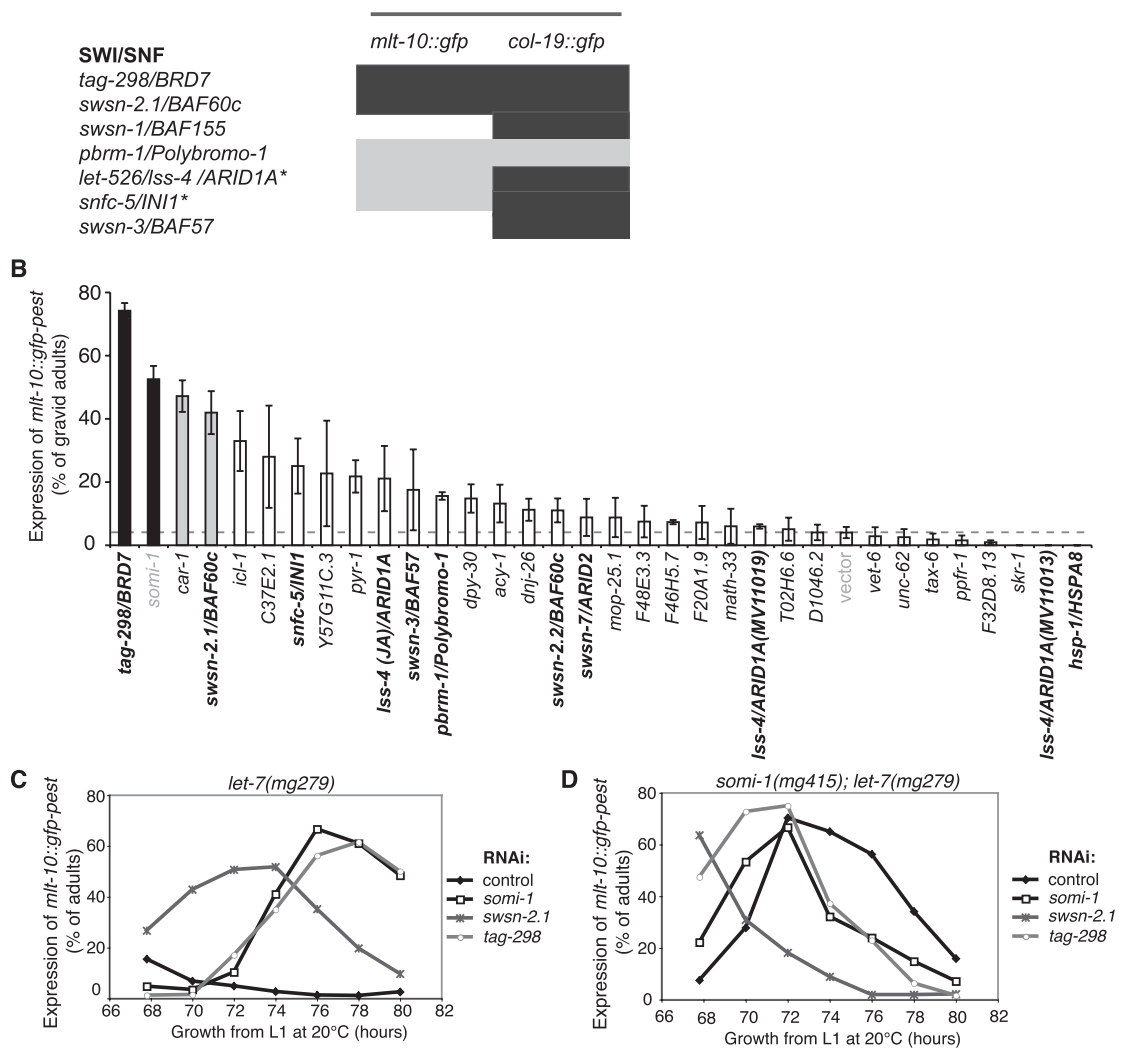

Figure 7. SWI/SNF-related proteins that copurify with SOMI-1 regulate differentiation of the hypodermis. (A) Many genes that enhance let-7(mg279) when inactivated encode homologs of components of the SWI/ SNF chromatin-remodeling complex. Human orthologs are given. Black and gray boxes indicate strong and weak enhancement, respectively. swsn-1 was not assayed for mlt$10:: g f p$ expression because its inactivation slowed development. The asterisk ( $\left.{ }^{\star}\right)$ denotes genes whose products did not copurify with SOMI-1 but were assayed because they have a related function. (B) Chart of data from Supplemental Table S2. Synchronized let7(mg279) L1 larvae were placed on the indicated RNAi clone and scored at $\sim 76 \mathrm{~h}$. The dashed line indicates percentage of gravid adults expressing mlt-10p::gfp-pest when treated with control bacteria. SWI/SNF-related gene names are bold, and somi-1 and vector controls are gray. Error bars represent SEM for independent experiments. Bar colors show significant difference from control: (black) $P<0.001$; (gray) $P<0.05$, one-way ANOVA, Tukey's post hoc test. $(C, D)$ Expression of mlt-10p::gfp-pest in the hypodermis of gravid GR1436 (C) or GR1605 (D) worms placed on the indicated RNAi bacteria as synchronized L1 larvae. At least 25 worms were scored at each time point. expression of mlt-10::gfp in the let-7(mg279) mutant (Fig. 7C; Supplemental Fig. S6B). To ask whether these SWI/SNF components require somi-1 to inhibit the supernumerary molt, we inactivated tag-298 and swsn-2.1 in a somi1(mg415); let-7(mg279) mutant background. We observed further enhancement (Fig. 7D; Supplemental Fig. S6C), indicating that SWI/SNF acts at least in part independently of SOMI-1 to inhibit the supernumerary molt. Consistent with this, an mCherry::TAG-298 fusion protein partly colocalized with SOMI-1::GFP foci in hypodermal nuclei but was also in parts of nuclei that lacked SOMI-1::GFP (Supplemental Fig. S8).

Finally, inactivation of unc-62, ceh-20, or ceh-40, which encode cofactors of homeobox (Hox) transcription factors (Van Auken et al. 2002), strongly restored expression of col-19::gfp in let-7 mir-84 or somi-1; let-7 adults (Supplemental Tables S2, S3; Supplemental Fig. S9). Inactivation of three of 46 additional homeodomain genes that we screened by RNAi-ceh-6/POU3F3, ceh-12/MNX1, and ceh-13/HOXB1-restored expression of col-19::gfp to the hypodermis of both let-7 mir-84 and somi-1; let-7 adults (Supplemental Table S3). Only a single peptide corresponding to UNC-62 suggested physical interaction of SOMI-1 with these factors. Thus, somi-1 may oppose the activity of Hox complexes that promote larval fates in the hypodermis, but this regulation may be indirect. Consistent with this possibility, inactivation of sop-2 or sor-1, which globally repress Hox gene expression, enhances the lethal bursting phenotype of let-7(mg279) (Zhang et al. 2006; Parry et al. 2007).

\section{Discussion}

Enrichment of SOMI-1 at the promoters of lin-14, lin-28, and let-60/RAS, transcripts of which may be targets of let-7 and mir-84, and the requirement for somi-1 for the defects caused by misexpression of mir-84 and let-7 suggest that somi-1 mediates a nuclear response to the activity of these miRNAs. Genetic and ChIP evidence suggest that SOMI-1 inhibits lin-14 and let-60/RAS in a way that complements their regulation by miRNAs (Supplemental Fig. S10). The identification of SOMI-1 and its interactors gives insight into how cytoplasmic inhibition of the target mRNAs of let-7 and its paralogs is translated into changes in chromatin structure and gene expression that promote differentiation. somi-1 provides competence to respond to mir-84: Like deletion of mir-84, somi-1-null mutations alone cause no dramatic defects in developmental timing, but somi-1 is required for the defects caused by elevated expression of mir-84, and its loss phenocopies the developmental timing deficits in a let-7 mutant background conferred by loss of mir-84.

let-7 promotes differentiation of the hypodermis in part by inhibiting lin-41 to allow the function of LIN-29, which effects a transcriptional switch from larval to adult components of the cuticle (Rougvie and Ambros 1995; Slack et al. 2000). The fact that somi-1 acts with let-7 to promote full expression of the adult-specific collagen gene col-19, transcription of which is activated by LIN-29, means that somi-1 acts at least in part in the canonical heterochronic 
pathway. But the identification of targets of let-7 in addition to lin-41 (Abrahante et al. 2003; Lin et al. 2003; Grosshans et al. 2005) and the fact that overexpression of mir-84 partly suppresses the supernumerary molting phenotype of a lin-29-null mutant (Hayes et al. 2006) indicate that factors in addition to LIN-29 promote differentiation of the hypodermis and exit from the molting cycle.

A small number of loci facilitate the function of mir-84. Our screen recovered three alleles of somi-1, about onesixth of the mutants found in the screen of the $\mathrm{F}_{2}$ progeny of the mutagenized worms. In addition, in a screen of the RNAi clones from chromosome four, about one-sixth of the genome, none robustly enabled egg laying in mir-84overexpressing animals (Hayes and Ruvkun 2005). It is unlikely that this is because RNAi is ineffective in generating the Somi phenotype, because somi-1 loss of function is phenocopied well by RNAi. Thus, identification of a small number of additional Somi mutants may facilitate a relatively complete picture of how mir-84 regulates development.

\section{Molecular function of SOMI-1}

A molecular function of SOMI-1 is suggested by its identity as a zinc finger protein, its localization to nuclear foci, and its enrichment at particular loci. Many zinc finger proteins are transcription factors, although the zinc finger may also mediate interaction with RNA or proteins (Laity et al. 2001). Some proteins with a single zinc finger, such as SUPERMAN in Arabidopsis and the Trithorax-like GAGA factor in Drosophila, bind specific DNA sequences (Dathan et al. 2002), meaning that SOMI1 could target other factors to particular loci. The nuclear foci formed by SOMI-1::GFP likely reflect endogenous SOMI-1 function, because SOMI-1::GFP rescues somi-1 mutant defects. C. elegans HPL-2, a homolog of heterochromatin protein 1 (HP1) that inhibits vulval induction, is recruited to nuclear foci by the zinc finger transcription factor LIN-13 (Coustham et al. 2006). SOP-2 and SOR-1, which form a Polycomb-like complex that represses Hox genes, also localize to nuclear foci (Zhang et al. 2006). Although somi-1 may oppose the function of some homeodomain genes, loss of somi-1, unlike sop-2 or sor-1, does not globally derepress Hox gene expression (data not shown). However, localization of SOMI-1 to nuclear foci and its copurification with SWI/SNF-related proteins suggest that it may promote chromatin remodeling to inhibit expression of particular target genes of mir-84.

\section{Chromatin-remodeling complexes and differentiation}

It is increasingly clear that not only are new DNA sequence-specific transcription factors expressed during tissue differentiation, but the composition of general transcription factor complexes such as TFIID, Mediator, and SWI/SNF changes as well (D'Alessio et al. 2009). For example, the SWI/SNF subunits Baf45a and Baf53a act in neural progenitors of mice, but their replacement with Baf $45 \mathrm{~b}$ and Baf53b drives differentiation into post-mitotic neurons (Lessard et al. 2007). This switch is promoted by miRNAs mir-9* and mir-124 (Yoo et al. 2009).
Our results, together with prior identification of 1ss-4/ ARID1 as a target of let-7 (Grosshans et al. 2005), suggest that let-7 could promote a similar shift from LSS-4-containing BAF-like complexes to PBAF-like complexes containing PBRM-1, TAG-298/BRD7, and SWSN-2.1/BAF60c that act with SOMI-1 to promote differentiation of the hypodermis. Arid1a (the ortholog of LSS-4) and Arid2 are diminished compared with other SWI/SNF components upon differentiation of mouse embryonic stem cells (Kaeser et al. 2008), suggesting that down-regulation of LSS-4/ Aridla upon differentiation of particular tissues may be conserved. LSS-4 did not copurify with SOMI-1::GFP, in contrast to PBAF-specific subunits PBRM-1/Polybromo, TAG-298/BRD7, and SWSN-7/ARID2 (Kwon and Wagner 2007; Kaeser et al. 2008). Furthermore, three different lss-4 RNAi clones caused the majority of $1 e t-7(\mathrm{mg} 279)$ mutants to burst at the vulva, an effect that we observed for no other SWI/SNF component nor somi-1 (Supplemental Table S2). This may seem to contradict the identification of 1 ss-4 as a suppressor of let-7 (Grosshans et al. 2005), but both the activity of $1 s s-4$ during larval growth and its down-regulation in adults may be needed for normal development. Our RNAi conditions may have more strongly inactivated $1 s s-4$ at the time of vulval specification. Unique association of the bursting phenotype with 1ss-4(RNAi) and absence of LSS-4 from the SOMI-1::GFP purification are consistent with SOMI-1 acting specifically with PBAF-like complexes to promote differentiation.

Vertebrate orthologs of the PBAF subunits that act with somi-1 and let-7 to promote differentiation of the hypodermis have analogous roles. The human ortholog of TAG298, BRD7, is a candidate tumor suppressor required for p53-mediated senescence and potentially other differentiation events (Burrows et al. 2010; Drost et al. 2010). BAF60c, the vertebrate ortholog of SWSN-2.1, mediates interactions between BAF complexes and transcription factors (Debril et al. 2004) and promotes skeletal muscle differentiation (Simone et al. 2004). Regulation of the vertebrate ortholog of LIN-41 by let-7 is proposed to control muscle differentiation (Lancman et al. 2005), raising the possibility that let-7 and BAF60c cooperate in this process.

SWI/SNF complexes in the balance appear, like somi-1, to inhibit VPC induction. Worms mutant for swsn-1, which encodes a core component of all SWI/SNF complexes, have an impenetrant Muv phenotype that is synergistically enhanced by mutation of lin-15, an inhibitor of lin-3/EGF expression (Cui et al. 2004). In contrast, mutations in several SWI/SNF accessory subunits impair vulval induction (Cui et al. 2006; Lehner et al. 2006). Mutations in accessory subunits may both positively and negatively affect transcription of various target genes, as observed for inactivation of the orthologs of $1 s s-4$ and tag-298 in mammalian cells (Kaeser et al. 2008).

In C. elegans, SWI/SNF complexes act with several signaling pathways to control the fate of hypodermal cells. For example, the larval asymmetric division of the $\mathrm{T}$ cell, the most posterior of the seam cells, which differentiate under the control of let-7 at the adult stage, is regulated by SWI/SNF, together with Wnt signaling (Sawa et al. 2000), the Hox protein NOB-1/Abd, and cofactors 
PSA-3/Meis and CEH-20/Pbx (Arata et al. 2006), and the nuclear hormone receptor encoded by $n h r-25$ (Hajduskova et al. 2009), itself a target of let-7 (Hayes et al. 2006). SOMI1 may promote alterations in chromatin structure, mediated by a PBAF-like complex, that occur in response to expression of mir-84 and let-7 and oppose the activity of larval fate-promoting factors.

somi-1 may regulate several aspects of vulval development

We showed here that somi-1 interacts genetically or physically with regulators of vulval development. One or more of these interactions may explain why loss of somi-1 suppresses the precocious vulval development that results when mir-84 is overexpressed. Activation of a let-60/RAS-MAP kinase cascade in the P6.p cell by expression of 7 in-3/EGF in the adjacent anchor cell during the L3 stage specifies $1^{\circ}$ vulval fate. RAS signaling also inhibits expression of lin-12/Notch in P6.p, establishing lateral inhibition that specifies $2^{\circ}$ vulval fate in the neighboring VPCs (Sternberg 2005). Given that mir-84 targets let-60, elevated expression of mir-84 might be expected to cause a let-60(lf) phenotype. However, let-60 hypomorphs are vulvaless, due to lack of the inductive signal (Beitel et al. 1990). The precocious divisions of the VPCs and Evl phenotype of mir-84-overexpressing animals are more like those of lin-14 and lin-28 mutants. lin-14 and lin-28 control the timing of re-entry of the VPCs into the cell cycle, thus regulating when they can respond to signals that specify cell fate (Euling and Ambros 1996; Ambros 1999; Li and Greenwald 2010). SWI/SNF proteins also have a role in vulva formation. The simplest hypothesis is that mir-84 overexpression causes precocious down-regulation of lin14 in the VPCs, which is suppressed by up-regulation of lin-14 in a somi-1 mutant. This is consistent with suppression of lin-14(lf) mutations by inactivation of somi-1. Given the binding of SOMI-1 to the promoters of lin-14, lin-28, and let-60/RAS and its physical interaction with SWI/SNF components, somi-1 may influence vulval development at multiple levels.

\section{Functions of paralogous miRNAs}

Many miRNAs are members of paralogous families, which may redundantly regulate the same or related target mRNAs (Lau et al. 2001; Miska et al. 2007). In some cases, loss of single members of paralogous families has yielded phenotypes (Reinhart et al. 2000), but deletion of paralogs of let-7, for example, caused obvious defects only in animals lacking two or more of these miRNAs (Abbott et al. 2005). Overexpression of particular miRNAs can reveal the function of members of paralogous families: Overexpression of lin-4 or let-7 causes precocious heterochronic phenotypes the opposite of those caused by a lossof-function mutation of each miRNA (Feinbaum and Ambros 1999; Reinhart et al. 2000). Similarly, apoptosis was inhibited by increased expression of the bantam miRNA in Drosophila, but was promoted by deletion of bantam (Brennecke et al. 2003). One concern is that offtarget effects could result if an overexpressed miRNA binds to target sequences it would not regulate at physiological levels of expression. Yet the phenotypes caused by overexpression of mir-84 (precocious development of hypodermal and vulval tissues) are consistent with loss-offunction phenotypes of genes in the heterochronic pathway, in which let-7 and its paralogs act. Genetic screens for suppression of the phenotypes conferred by overexpression of miRNAs can also identify components of the pathways in which they act.

somi-1 is required for the precocious phenotypes that result when mir-84 is overexpressed, but is dispensable for similar defects caused by elevated expression of mir48. This suggests that these paralogous miRNAs, identical in their 5" "seed" sequences and both expressed in the VPCs and seam cells during larval development (Li et al. 2005; Hayes et al. 2006), may recognize at least partly distinct sets of targets even when overexpressed. The miRNA 5' seed sequence is proposed to be most important in target selection (Doench and Sharp 2004), but complementarity of the $3^{\prime}$ end of let-7 to its target sites is important for regulation of lin-41 (Vella et al. 2004), and additional determinants influence target site function (Didiano and Hobert 2006; Bartel 2009).

\section{Materials and methods}

\section{Mutagenesis screen}

C. elegans of genotype mgIs45[mir-84++] I; wIs54[scm::gfp] V were mutagenized with ethylmethane sulfonate. Rare eggs laid by the progeny of $\sim 3700 \mathrm{~F}_{1}$ animals were picked to new plates to establish putative Somi mutant strains. Male tail defects caused by overexpression of mir-84 (data not shown) hampered assignment of the Somi isolates to complementation groups.

\section{Rescue of somi-1}

An 8-kb fragment containing $5 \mathrm{~kb}$ of M04G12.4 coding sequence and predicted $3^{\prime}$ UTR and $3 \mathrm{~kb}$ of sequence upstream was amplified from C. elegans genomic DNA with Expand High-Fidelity polymerase (Roche) and primers GH183 5'-caacccgggGCAGAA AATTAGGTCTGGAATCG-3' (sequence homologous to somi-1 is capitalized) and GH176 5' -TACTGGGCGGTCATATGTTC-3'. Three separate PCRs were pooled for injections at $0.5 \mathrm{ng} / \mu \mathrm{L}$ with plasmid specifying $t t x-3:: d s R e d$ (courtesy of Ho Yi Mak) at $50 \mathrm{ng} /$ $\mu \mathrm{L}$, yielding transgene $m g E x 740$, which was crossed into strain GR1576 to create strain GR1579 mgEx740[somi-1(+)]; mgIs45 [mir-84++] I; somi-1(mg415) V.

\section{Assay for egg laying defects}

L4 larvae or adults that formed a clear vulval protrusion were scored as Evl. Otherwise, animals were moved singly to plates and typically scored the next day for ability to lay eggs. Those unable to lay eggs were scored as Evl. In RNAi experiments, animals grown for two generations on RNAi bacteria were moved as adults to plates seeded with strain OP50 for scoring the Evl phenotype. Infertile animals were not scored.

\section{Microscopy}

Images were captured on a Zeiss Axioplan microscope using a Hamamatsu ORCA-ER digital camera and Openlab (Improvision) 
Hayes et al.

or Axiovision (Zeiss) software. Deconvolution was done with Axiovision.

\section{RNAi}

RNAi was performed essentially as described by Fraser et al. (2000) and modified by Hayes et al. (2006), where the lin-28 RNAi clone is described. Typically, L4 larvae were placed on RNAi bacteria and their $F_{1}$ progeny were scored. Control bacteria expressed dsRNA homologous to no worm gene.

ChIP

In brief, worms were frozen and ground under liquid nitrogen. The resulting powder was cross-linked using $1 \%$ formaldehyde in PBS and sonicated. SOMI-1::GFP was immunoprecipitated with polyclonal rabbit- $\alpha$-GFP antibody (Clontech) and Protein A Dynal beads (Invitrogen). Protein-DNA complexes were eluted and treated with RNase A and proteinase K. DNA was purified with a ChIP DNA purification kit (Zymo Research) and analyzed by qPCR.

\section{Acknowledgments}

We thank members of the Ruvkun, Kaplan, and Ausubel laboratories for comments during this project and critical reading of the manuscript, in particular X. Wu, S. Curran, A. Frand, H. Gabel, J. Kim, W. Li, H.Y. Mak, J. Melo, D. Parry, and S. Russel. We thank John Asara of Beth Israel Deaconess Medical Center for mass spectrometry, J. Xu for microinjections, and the Caenorhabditis Genetics Center at the University of Minnesota for strains. Ann Rougvie provided strains and helpful comments. somi-1(tm562) was isolated by the Mitani laboratory of the Japanese National BioResources Project. C.G.R. was supported by long-term fellowships from the European Molecular Biology Organization and the Human Frontier Science Program. This work was funded by NIH grant GM44619 to G.R.

\section{References}

Abbott AL, Alvarez-Saavedra E, Miska EA, Lau NC, Bartel DP, Horvitz HR, Ambros V. 2005. The let-7 microRNA family members mir-48, mir-84, and mir-241 function together to regulate developmental timing in Caenorhabditis elegans. Dev Cell 9: 403-414.

Abrahante JE, Miller EA, Rougvie AE. 1998. Identification of heterochronic mutants in Caenorhabditis elegans. Temporal misexpression of a collagen:green fluorescent protein fusion gene. Genetics 149: 1335-1351.

Abrahante JE, Daul AL, Li M, Volk ML, Tennessen JM, Miller EA, Rougvie AE. 2003. The Caenorhabditis elegans hunchback-like gene lin-57/hbl-1 controls developmental time and is regulated by microRNAs. Dev Cell 4: 625-637.

Alvarez-Garcia I, Miska EA. 2005. MicroRNA functions in animal development and human disease. Development 132: $4653-4662$.

Ambros V. 1999. Cell cycle-dependent sequencing of cell fate decisions in Caenorhabditis elegans vulva precursor cells. Development 126: 1947-1956.

Ambros V, Horvitz HR. 1984. Heterochronic mutants of the nematode Caenorhabditis elegans. Science 226: 409416.

Arata Y, Kouike H, Zhang Y, Herman MA, Okano H, Sawa H. 2006. Wnt signaling and a Hox protein cooperatively regulate psa-3/Meis to determine daughter cell fate after asymmetric cell division in C. elegans. Dev Cell 11: 105115.

Bagga S, Bracht J, Hunter S, Massirer K, Holtz J, Eachus R, Pasquinelli AE. 2005. Regulation by let-7 and lin-4 miRNAs results in target mRNA degradation. Cell 122: 553-563.

Bartel DP. 2009. MicroRNAs: target recognition and regulatory functions. Cell 136: 215-233.

Beitel GJ, Clark SG, Horvitz HR. 1990. Caenorhabditis elegans ras gene let-60 acts as a switch in the pathway of vulval induction. Nature 348: 503-509.

Bracht J, Hunter S, Eachus R, Weeks P, Pasquinelli AE. 2004. Trans-splicing and polyadenylation of let-7 microRNA primary transcripts. RNA 10: 1586-1594.

Brennecke J, Hipfner DR, Stark A, Russell RB, Cohen SM. 2003. bantam encodes a developmentally regulated microRNA that controls cell proliferation and regulates the proapoptotic gene hid in Drosophila. Cell 113: 25-36.

Burrows AE, Smogorzewska A, Elledge SJ. 2010. Polybromoassociated BRG1-associated factor components BRD7 and BAF180 are critical regulators of p53 required for induction of replicative senescence. Proc Natl Acad Sci 107: 1428014285.

Coustham V, Bedet C, Monier K, Schott S, Karali M, Palladino F. 2006. The C. elegans HP1 homologue HPL-2 and the LIN-13 zinc finger protein form a complex implicated in vulval development. Dev Biol 297: 308-322.

Cui M, Fay DS, Han M. 2004. lin-35/Rb cooperates with the SWI/SNF complex to control Caenorhabditis elegans larval development. Genetics 167: 1177-1185.

Cui M, Kim EB, Han M. 2006. Diverse chromatin remodeling genes antagonize the Rb-involved SynMuv pathways in C. elegans. PLoS Genet 2: e74. doi: 10.1371/journal/ pgen.0020074.

D'Alessio JA, Wright KJ, Tjian R. 2009. Shifting players and paradigms in cell-specific transcription. Mol Cell 36: 924931.

Dathan N, Zaccaro L, Esposito S, Isernia C, Omichinski JG, Riccio A, Pedone C, Di Blasio B, Fattorusso R, Pedone PV. 2002. The Arabidopsis SUPERMAN protein is able to specifically bind DNA through its single Cys2-His2 zinc finger motif. Nucleic Acids Res 30: 4945-4951.

Debril MB, Gelman L, Fayard E, Annicotte JS, Rocchi S, Auwerx J. 2004. Transcription factors and nuclear receptors interact with the SWI/SNF complex through the BAF60c subunit. J Biol Chem 279: 16677-16686.

Didiano D, Hobert O. 2006. Perfect seed pairing is not a generally reliable predictor for miRNA-target interactions. Nat Struct Mol Biol 13: 849-851.

Ding L, Spencer A, Morita K, Han M. 2005. The developmental timing regulator AIN-1 interacts with miRISCs and may target the argonaute protein ALG-1 to cytoplasmic P bodies in C. elegans. Mol Cell 19: 437-447.

Doench JG, Sharp PA. 2004. Specificity of microRNA target selection in translational repression. Genes Dev 18: 504511.

Drost J, Mantovani F, Tocco F, Elkon R, Comel A, Holstege H, Kerkhoven R, Jonkers J, Voorhoeve PM, Agami R, et al. 2010. $\mathrm{BRD} 7$ is a candidate tumour suppressor gene required for $\mathrm{p} 53$ function. Nat Cell Biol 12: 380-389.

Esquela-Kerscher A, Johnson SM, Bai L, Saito K, Partridge J, Reinert KL, Slack FJ. 2005. Post-embryonic expression of $C$. elegans microRNAs belonging to the lin-4 and let-7 families in the hypodermis and the reproductive system. Dev Dyn 234: $868-877$. 
Euling S, Ambros V. 1996. Heterochronic genes control cell cycle progress and developmental competence of C. elegans vulva precursor cells. Cell 84: 667-676.

Feinbaum R, Ambros V. 1999. The timing of lin-4 RNA accumulation controls the timing of postembryonic developmental events in Caenorhabditis elegans. Dev Biol 210: 87-95.

Frand AR, Russel S, Ruvkun G. 2005. Functional genomic analysis of C. elegans molting. PLoS Biol 3: e312. doi: 10.1371/journal. pbio.0030312.

Fraser AG, Kamath RS, Zipperlen P, Martinez-Campos M, Sohrmann M, Ahringer J. 2000. Functional genomic analysis of C. elegans chromosome I by systematic RNA interference. Nature 408: 325-330.

Grosshans H, Johnson T, Reinert KL, Gerstein M, Slack FJ. 2005. The temporal patterning microRNA let-7 regulates several transcription factors at the larval to adult transition in $C$. elegans. Dev Cell 8: 321-330.

Hajduskova M, Jindra M, Herman MA, Asahina M. 2009. The nuclear receptor NHR-25 cooperates with the Wnt/ $\beta$-catenin asymmetry pathway to control differentiation of the $\mathrm{T}$ seam cell in C. elegans. J Cell Sci 122: 3051-3060.

Hayes GD, Ruvkun G. 2005. "Control of developmental timing by microRNAs in C. elegans." PhD thesis. Harvard University, Cambridge, MA.

Hayes GD, Ruvkun G. 2006. Misexpression of the Caenorhabditis elegans miRNA let-7 is sufficient to drive developmental programs. Cold Spring Harb Symp Quant Biol 71: 21-27.

Hayes GD, Frand AR, Ruvkun G. 2006. The mir-84 and let-7 paralogous microRNA genes of Caenorhabditis elegans direct the cessation of molting via the conserved nuclear hormone receptors NHR-23 and NHR-25. Development 133: 4631-4641.

Hebert C, Norris K, Scheper MA, Nikitakis N, Sauk JJ. 2007. High mobility group A2 is a target for miRNA-98 in head and neck squamous cell carcinoma. Mol Cancer 6: 5 .

Johnson SM, Grosshans H, Shingara J, Byrom M, Jarvis R, Cheng A, Labourier E, Reinert K, Brown D, Slack FJ. 2005. RAS is regulated by the let- 7 microRNA family. Cell 120: 635-647.

Kaeser MD, Aslanian A, Dong MQ, Yates JR III, Emerson BM. 2008. BRD7, a novel PBAF-specific SWI/SNF subunit, is required for target gene activation and repression in embryonic stem cells. J Biol Chem 283: 32254-32263.

Kong YW, Cannell IG, de Moor CH, Hill K, Garside PG, Hamilton TL, Meijer HA, Dobbyn HC, Stoneley M, Spriggs $\mathrm{KA}$, et al. 2008. The mechanism of micro-RNA-mediated translation repression is determined by the promoter of the target gene. Proc Natl Acad Sci 105: 8866-8871.

Kwon CS, Wagner D. 2007. Unwinding chromatin for development and growth: a few genes at a time. Trends Genet 23: 403-412.

Laity JH, Lee BM, Wright PE. 2001. Zinc finger proteins: new insights into structural and functional diversity. Curr Opin Struct Biol 11: 39-46.

Lancman JJ, Caruccio NC, Harfe BD, Pasquinelli AE, Schageman JJ, Pertsemlidis A, Fallon JF. 2005. Analysis of the regulation of lin-41 during chick and mouse limb development. Dev Dyn 234: 948-960.

Lau NC, Lim LP, Weinstein EG, Bartel DP. 2001. An abundant class of tiny RNAs with probable regulatory roles in Caenorhabditis elegans. Science 294: 858-862.

Lee RC, Feinbaum RL, Ambros V. 1993. The C. elegans heterochronic gene lin-4 encodes small RNAs with antisense complementarity to lin-14. Cell 75: 843-854.
Lehner B, Crombie C, Tischler J, Fortunato A, Fraser AG. 2006. Systematic mapping of genetic interactions in Caenorhabditis elegans identifies common modifiers of diverse signaling pathways. Nat Genet 38: 896-903.

Lessard J, Wu JI, Ranish JA, Wan M, Winslow MM, Staahl BT, Wu H, Aebersold R, Graef IA, Crabtree GR. 2007. An essential switch in subunit composition of a chromatin remodeling complex during neural development. Neuron 55: 201-215.

Li J, Greenwald I. 2010. LIN-14 inhibition of LIN-12 contributes to precision and timing of C. elegans vulval fate patterning. Curr Biol 20: 1875-1879.

Li M, Jones-Rhoades MW, Lau NC, Bartel DP, Rougvie AE. 2005. Regulatory mutations of mir-48, a C. elegans let-7 family microRNA, cause developmental timing defects. Dev Cell 9: 415-422.

Lin SY, Johnson SM, Abraham M, Vella MC, Pasquinelli A, Gamberi C, Gottlieb E, Slack FJ. 2003. The C elegans hunchback homolog, hbl-1, controls temporal patterning and is a probable microRNA target. Dev Cell 4: 639-650.

Miska EA, Alvarez-Saavedra E, Abbott AL, Lau NC, Hellman AB, McGonagle SM, Bartel DP, Ambros VR, Horvitz HR. 2007. Most Caenorhabditis elegans microRNAs are individually not essential for development or viability. PLoS Genet 3: e215. doi: 10.1371/jounral.pgen.0030215.

Moshkin YM, Mohrmann L, van Ijcken WF, Verrijzer CP. 2007. Functional differentiation of SWI/SNF remodelers in transcription and cell cycle control. Mol Cell Biol 27: 651-661.

Moss EG, Lee RC, Ambros V. 1997. The cold shock domain protein LIN-28 controls developmental timing in C. elegans and is regulated by the lin-4 RNA. Cell 88: 637-646.

Nimmo RA, Slack FJ. 2009. An elegant miRror: microRNAs in stem cells, developmental timing and cancer. Chromosoma 118: 405-418.

Parry DH, Xu J, Ruvkun G. 2007. A whole-genome RNAi Screen for C. elegans miRNA pathway genes. Curr Biol 17: 2013-2022.

Pasquinelli AE, Reinhart BJ, Slack F, Martindale MQ, Kuroda MI, Maller B, Hayward DC, Ball EE, Degnan B, Muller P, et al. 2000. Conservation of the sequence and temporal expression of let-7 heterochronic regulatory RNA. Nature 408: $86-89$.

Reinhart BJ, Ruvkun G. 2001. Isoform-specific mutations in the Caenorhabditis elegans heterochronic gene lin-14 affect stage-specific patterning. Genetics 157: 199-209.

Reinhart BJ, Slack FJ, Basson M, Pasquinelli AE, Bettinger JC, Rougvie AE, Horvitz HR, Ruvkun G. 2000. The 21-nucleotide let-7 RNA regulates developmental timing in Caenorhabditis elegans. Nature 403: 901-906.

Rougvie AE, Ambros V. 1995. The heterochronic gene lin-29 encodes a zinc finger protein that controls a terminal differentiation event in Caenorhabditis elegans. Development 121: 2491-2500.

Sawa H, Kouike H, Okano H. 2000. Components of the SWI/ SNF complex are required for asymmetric cell division in $C$. elegans. Mol Cell 6: 617-624.

Simone C, Forcales SV, Hill DA, Imbalzano AN, Latella L, Puri PL. 2004. p38 pathway targets SWI-SNF chromatin-remodeling complex to muscle-specific loci. Nat Genet 36: 738-743.

Slack FJ, Basson M, Liu Z, Ambros V, Horvitz HR, Ruvkun G. 2000. The lin-41 RBCC gene acts in the C. elegans heterochronic pathway between the let-7 regulatory RNA and the LIN-29 transcription factor. Mol Cell 5: 659-669.

Sternberg PW. 2005. Vulval development. WormBook (ed. The C. elegans Research Community), WormBook, doi: 10.1895/ wormbook.1.6.1. http://www.wormbook.org. 
Hayes et al.

Van Auken K, Weaver D, Robertson B, Sundaram M, Saldi T, Edgar L, Elling U, Lee M, Boese Q, Wood WB. 2002. Roles of the Homothorax/Meis/Prep homolog UNC-62 and the Exd/ $\mathrm{Pbx}$ homologs $\mathrm{CEH}-20$ and $\mathrm{CEH}-40$ in C. elegans embryogenesis. Development 129: 5255-5268.

Vella MC, Reinert K, Slack FJ. 2004. Architecture of a validated microRNA:target interaction. Chem Biol 11: 1619-1623.

Wightman B, Ha I, Ruvkun G. 1993. Posttranscriptional regulation of the heterochronic gene lin-14 by lin- 4 mediates temporal pattern formation in C. elegans. Cell 75: 855-862.

Yoo AS, Staahl BT, Chen L, Crabtree GR. 2009. MicroRNAmediated switching of chromatin-remodelling complexes in neural development. Nature 460: 642-646.

Zhang T, Sun Y, Tian E, Deng H, Zhang Y, Luo X, Cai Q, Wang H, Chai J, Zhang H. 2006. RNA-binding proteins SOP-2 and SOR-1 form a novel PcG-like complex in C. elegans. Development 133: 1023-1033. 


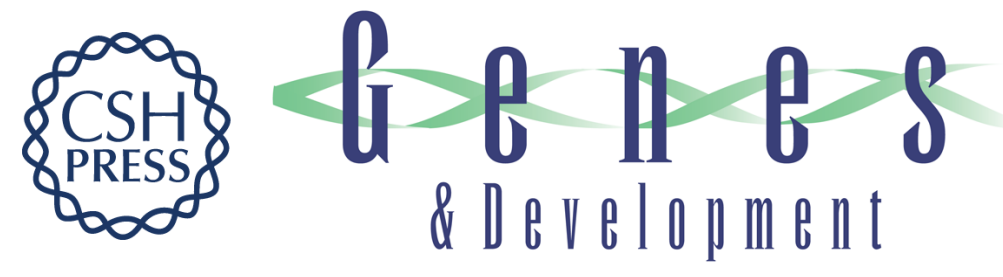

\section{The Caenorhabditis elegans SOMI-1 zinc finger protein and SWI/SNF promote regulation of development by the mir-84 microRNA}

Gabriel D. Hayes, Christian G. Riedel and Gary Ruvkun

Genes Dev. 2011, 25:

Access the most recent version at doi:10.1101/gad.17153811

Supplemental http://genesdev.cshlp.org/content/suppl/2011/10/06/25.19.2079.DC1
Material

References This article cites 63 articles, 16 of which can be accessed free at:

http://genesdev.cshlp.org/content/25/19/2079.full.html\#ref-list-1

License

Email Alerting

Receive free email alerts when new articles cite this article - sign up in the box at the top

Service

right corner of the article or click here.

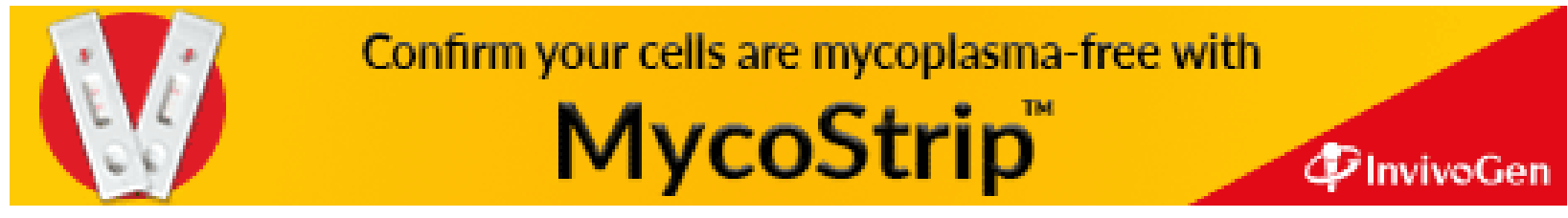

